\title{
Theoretical Analysis and Experimental Validation of a Simplified Fractional Order Controller for a Magnetic Levitation System
}

\author{
Silviu Folea, Member, IEEE, Cristina I. Muresan, Robin De Keyser, and Clara M. Ionescu, Member, IEEE
}

\begin{abstract}
Fractional order (FO) controllers are among the emerging solutions for increasing closed-loop performance and robustness. However, they have been applied mostly to stable processes. When applied to unstable systems, the tuning technique uses the well-known frequency-domain procedures or complex genetic algorithms. This brief proposes a special type of an FO controller, as well as a novel tuning procedure, which is simple and does not involve any optimization routines. The controller parameters may be determined directly using overshoot requirements and the study of the stability of FO systems. The tuning procedure is given for the general case of a class of unstable systems with pole multiplicity. The advantage of the proposed FO controller consists in the simplicity of the tuning approach. The case study considered in this brief consists in a magnetic levitation system. The experimental results provided show that the designed controller can indeed stabilize the magnetic levitation system, as well as provide robustness to modeling uncertainties and supplementary loading conditions. For comparison purposes, a simple PID controller is also designed to point out the advantages of using the proposed FO controller.
\end{abstract}

Index Terms - Control design, fractional calculus, robustness, stability analysis, unstable systems.

\section{INTRODUCTION}

$\mathbf{T}$ HE control of magnetic levitation systems has received considerable scientific interest not only because of the highly nonlinear and unstable behavior of such systems [1] but also because of their ability to eliminate friction, decrease maintenance cost, and achieve high-precision positioning [1], [2]. These advantages make magnetic levitation systems a viable choice for high-speed trains, magnetic bearings, vibration isolation systems, and wind tunnel levitation [1], [3], [4].

Numerous control solutions have been proposed for such systems, such as feedback linearization techniques [5], which

Manuscript received October 27, 2014; revised March 2, 2015; accepted May 3, 2015. Date of publication July 8, 2015; date of current version February 17, 2016. Manuscript received in final form May 21, 2015. This work was supported by the Romanian National Authority for Scientific Research National Council for Development and Innovation-Executive Unit for Financing Higher Education, Research, Development and Innovation under Project PN-II-RU-TE-2012-3-0307 and Project TE 59/2013. Recommended by Associate Editor Y. Chen.

S. Folea, C. I. Muresan, and C. M. Ionescu are with the Department of Automation, Technical University of Cluj-Napoca, Cluj-Napoca 400604, Romania (e-mail: silviu.folea@aut.utcluj.ro; cristina.pop@aut.utcluj.ro; claramihaela.ionescu@ugent.be).

R. De Keyser is with the Department of Electrical Energy, Systems and Automation, Ghent University, Ghent 9052, Belgium (e-mail: robain.dekeyser@ugent.be).

Color versions of one or more of the figures in this paper are available online at http://ieeexplore.ieee.org.

Digital Object Identifier 10.1109/TCST.2015.2446496 require a very accurate model for the magnetic levitation system. This may represent a major problem, since a precise dynamic model may be difficult to obtain [1], [5] because of the prevailing nonlinearities that characterize the system, including the variation of the gain of the magnetic levitation system as a function of the distance to the magnet [6]. Other control approaches are based on designing the controllers for the linearized dynamic model at nominal operating points. Nevertheless, the tracking performances of such control strategies deteriorate drastically with increasing deviation from nominal operating points [1]. To account for the changing parameters and dynamics of magnetic levitation systems, sliding mode, nonlinear, $\mu$-synthesis, PIDs combined with notch filters, gain scheduling, backstepping, and fuzzy neural network-based controllers have been proposed, providing robustness against unmodeled nonlinearities present in the system [1], [3], [5]-[12]. A networked control system application on an unstable triple-magnetic-levitation setup has also been reported, with the controllers tuned according to the $H_{\infty}$ theory [13].

Fractional calculus has been listed among the current trends in control engineering, with a wide application in both modeling [14], [15] and control design [16]-[19]. The main advantage of fractional order (FO) controllers is their ability to enhance the performance of closed-loop systems and increase the robustness [6], [19], [20]. The tuning approach for $\mathrm{FO} \mathrm{PI}^{\lambda} \mathrm{D}^{\mu}$ controllers consists in specifying a set of performance criteria and transposing these specifications into several equations. Using optimization routines or graphical methods the controller parameters are determined [21]-[23]. For unstable systems, few papers deal with the design of FO controllers [24], [25]. For magnetic levitation systems, FO controllers have been previously designed [26], [27]. However, all of these papers propose the general form of the FO PI or PD controllers and the tuning technique uses the well-known frequency-domain procedures [27] or complex genetic algorithms [26].

In this brief, a special type of an FO controller, different than the usual FO PID, is proposed for the magnetic levitation system. The tuning procedure is simple and does not involve any optimization routines. The controller parameters may be determined directly using overshoot requirements and the study of the stability of FO systems. The advantage of the proposed FO controller consists in the simplicity of the tuning approach. The novelty of this brief resides in the new tuning technique for FO controllers, as well as in the different form of the FO controller proposed for a class of unstable systems 
with pole multiplicity. In addition, this brief presents the implementation and experimental validation of the proposed control algorithm on a pilot scale magnetic levitation stand. An analysis regarding the robustness of the proposed controller is also included.

This brief is structured into five main parts. Section II presents the algorithm for tuning stabilizing FO controllers for a set of unstable systems with pole multiplicity. Section III details the experimental setup, as well as the controller tuning procedure and the experimental results. The robustness analysis is carried out in Section IV, including several operating points, as well as two case studies in which supplementary loads are added to the permanent disk magnet. The experimental results show that the designed controller is robust against modeling uncertainties and intrinsic nonlinearities. Section V presents the comparison of the proposed control strategy with a simple PID controller, while the final section summarizes the main outcome of this brief.

\section{StABILITy ANALYSis OF A FRACTIONAL ORDER}

\section{CONTROLler For a Class of Unstable Processes}

The transfer function of the class of unstable systems with pole multiplicity considered in this brief is

$$
G(s)=\frac{1}{(s-z)(s+z)}
$$

with $z>0$. A simple PD controller may be designed to stabilize the system

$$
C_{\mathrm{PD}}(s)=k(s+z)
$$

with $z$ chosen to compensate the stable pole of the system in (1). The resulting closed-loop system will exhibit an overdamped response, for a value of the gain $k_{\text {stab }}$ that will make the system stable. However, such a PD controller will exhibit steady-state offsets, which could be eliminated with a simple PID controller. An alternative solution to the classical integer order PID controller is proposed in this brief, consisting in a PD controller and a fractional integrator that eliminates the steady-state errors

$$
C(s)=k \frac{(s+z)}{s^{\lambda}}
$$

with $\lambda \in[0,1]$. For certain values of $k$ and $\lambda$, the closed-loop system will become stable. The choice of the FO controller in (3), apart from its simplicity, is also justified by the lower number of tuning parameters. The controller in (3) may be rewritten as an FO $I^{\lambda} D^{1-\lambda}$ controller

$$
C(s)=k\left(s^{1-\lambda}+\frac{z}{s^{\lambda}}\right) .
$$

The open-loop transfer function would then be given as

$$
H_{\text {open-loop }}(s)=C(s) G(s)=\frac{k}{s^{\lambda}(s-z)} .
$$

The characteristic equation used to determine the stability of the closed-loop system is then

$$
k+s^{\lambda}(s-z)=0 .
$$

The equation in (6) is a function of the complex variable $s$ whose domain can be seen as a Riemann surface, with the principal sheet defined as $-\pi<\arg (s)<\pi$ [22]. This definition of the principal sheet assumes a cut along $R^{-}$and is associated to the Cauchy principal value of the integral corresponding to the inverse transformation of Laplace, or to that obtained by direct application of the residue theorem [22]. In the case of $\lambda=(1 / n)$, where $n$ is a positive integer, the $n$ sheets of the Riemann surface will be given by

$$
s=|s| e^{j \phi},(2 i+1) \pi<\phi<(2 i+3) \pi
$$

with $i=-1,0,1, \ldots, n-2$. For example, for $i=-1$, the principal Riemann sheet is obtained, whereas for $i=0$, the secondary Riemann sheet is obtained. If a mapping is used, defined as $w=s^{\lambda}$, then the Riemann sheets become the regions in the $w$ plane defined by

$$
w=|w| e^{j \theta}, \quad \lambda(2 i+1) \pi<\theta<\lambda(2 i+3) \pi .
$$

The characteristic equation in (6) will have an infinite number of roots, among which only a finite number of roots will be on the principal sheet of the Riemann surface [28]. These roots are generally referred to as the structural roots of the system and are responsible for the closed-loop system exhibiting either damped oscillation, oscillation of constant amplitude, oscillation of increasing amplitude with monotonic growth. The roots that are located in the secondary sheets of the Riemann surface are related to solutions that are always monotonically decreasing functions [22], thus a system that has all poles in the secondary Riemann sheets is closed-loop stable.

For the case of commensurate-order systems, where all the orders of derivation are integer multiples of a base order, the characteristic equation is a polynomial of the complex variable $w=s^{\lambda}$. The stability condition for these types of systems is expressed as [22], [28], [29]

$$
|\theta|>\lambda \frac{\pi}{2} \text {. }
$$

Using (8) and making $i=-1$, the condition for the principal Riemann sheet is obtained as

$$
-\lambda \pi<\theta<\lambda \pi \text {. }
$$

Intersecting (9) and (10), yields the final stability condition for all roots lying in the first Riemann sheet

$$
\theta \epsilon\left(\lambda \frac{\pi}{2}, \lambda \pi\right) \cup\left(-\lambda \pi,-\lambda \frac{\pi}{2}\right) .
$$

In the case of $\lambda=(1 / n)$, where $n$ is a positive integer, the stability condition in (11) is modified to be

$$
\theta \epsilon\left(\frac{\pi}{2 n}, \frac{\pi}{n}\right) \cup\left(-\frac{\pi}{n},-\frac{\pi}{2 n}\right) .
$$

Considering the above mapping, $w=s^{\lambda}$, and $\lambda=(1 / n)$, the characteristic polynomial in (6) becomes

$$
w^{n+1}-z * w^{1}+k=0 .
$$

The tuning of the controller parameters $k$ and $\lambda$ is performed to meet the overshoot requirements (\%OS) and it is based on 


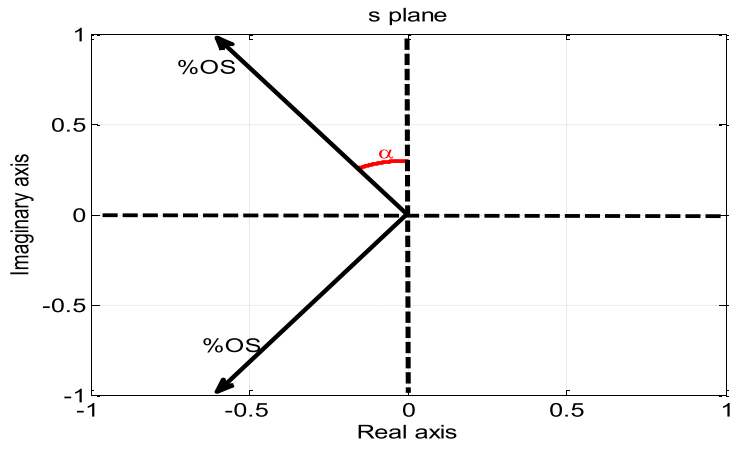

(a)

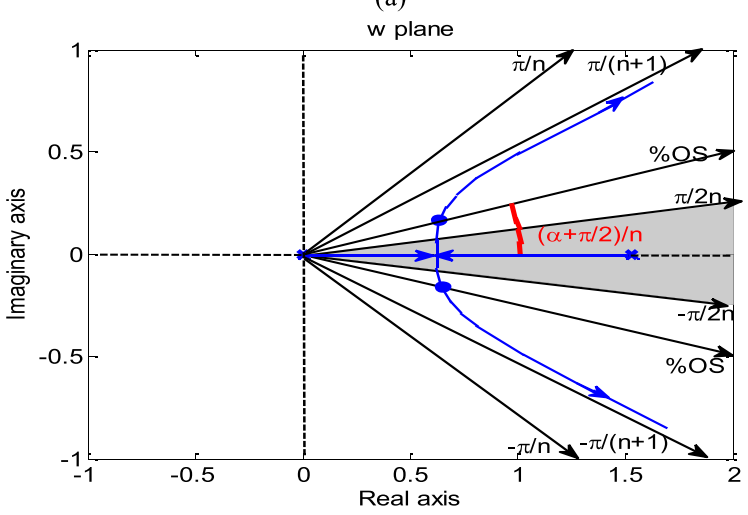

(b)

Fig. 1. Equivalence between (a) $s$ plane and (b) $w$ plane.

the root locus analysis of (13) in the $w$ plane. The equivalence of the $s$ and $w$ planes is given in Fig. 1. For $k=0$, the roots of (13) are

$$
w=0 \quad \text { and } \quad w=z^{\frac{1}{n}} e^{\frac{2 m \pi}{n}}
$$

with $m \in \mathbb{Z}$. It can be easily seen from (14) that for $m=0$, the roots that lie in the principal Riemann sheet correspond to $w=0$ and $w=z^{(1 / n)}$, with all other roots, corresponding to $m \neq 0$, located in the secondary sheets. The root locus corresponding to the two roots located in the principal Riemann sheet is given in Fig. 1(b). According to Fig. 1(a), in order for the closed-loop system to have a certain overshoot, denoted \%OS, the poles must lie on the \%OS line. The following relations hold:

$$
\sin \alpha=\zeta \text { and } \% \mathrm{OS}=e^{-\pi \xi / \sqrt{1-\zeta^{2}}}
$$

where $\xi$ is the damping factor. The \%OS line and its corresponding angle $\alpha$ in the $s$ plane are translated into the $w$ plane as indicated in Fig. 1(b), with the angle $\beta=((\alpha+(\pi / 2)) / n)$. In order for the closed-loop poles to have an exact overshoot \%OS, the angle of the asymptotes and the angle $\beta$ must meet the following requirement:

$$
\beta<\frac{\pi}{n+1} \text { or } \frac{\alpha+\frac{\pi}{2}}{n}<\frac{\pi}{n+1} .
$$

Using (15) and (16), a simple tuning procedure may be derived to meet an imposed overshoot \%OS*.

1) Compute damping factor $\xi$ as

$$
\zeta=\frac{\left|\ln \left(\% \mathrm{OS}^{*}\right)\right|}{\sqrt{\pi^{2}}+\ln ^{2}\left(\% \mathrm{OS}^{*}\right)} .
$$

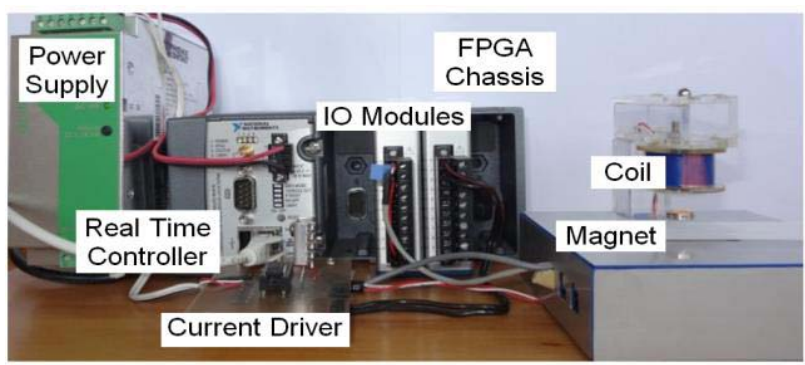

(a)

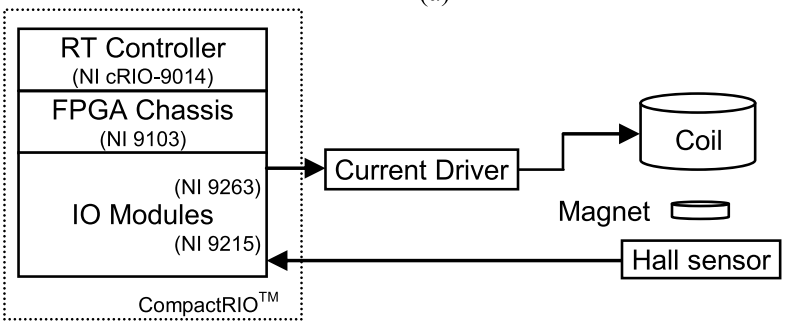

(b)

Fig. 2. (a) Experimental setup of the magnetic levitation system. (b) Block diagram of the magnetic levitation system.

2) Evaluate the angle $\alpha$, corresponding to the imposed $\% \mathrm{OS}^{*}$

$$
\alpha=\sin ^{-1} \xi .
$$

3) Using (16), determine the range for $n$ and the corresponding FO $\lambda$

$$
n>\frac{\frac{\pi}{2}+\alpha}{\frac{\pi}{2}-\alpha}
$$

Based on (17)-(19), for a small overshoot, $\alpha \rightarrow(\pi / 2)$, which in turn leads to $n \rightarrow \infty \Rightarrow \lambda \rightarrow 0$. Select $n$ as the minimum $n_{\min }$ that obeys (19).

4) Based on stability analysis in the $w$ plane, determine $k$ such that the roots of (13) located in the principal Riemann sheet lie on the imposed \%OS* line.

\section{TUNING AND EXPERIMENTAL VALIDATION OF A SIMPLIFIED FRACTIONAL ORDER CONTROLLER FOR A MAGNETIC LEVITATION SySTEM}

An experimental laboratory scale magnetic levitation unit has been developed and built, as given in Fig. 2(a), consisting of a small permanent disk magnet suspended in a voltage-controlled magnetic field. Several modules are used to implement the control algorithm, as indicated in Fig. 2(b), such as the NI cRIO-9014 embedded real-time controller, the NI 9103 reconfigurable chassis, and the NI 9215/NI 9263 input/output modules. The vertical position of the levitating permanent magnet is measured using an SS495A ratiometric linear Hall sensor, which generates an output voltage that is proportional with the magnetic field. The sensor is placed at the bottom of the experimental unit, away from the coil to eliminate the influence of the magnetic field generated by the coil on the magnetic field generated by the permanent magnet. A low-pass filter has also been 


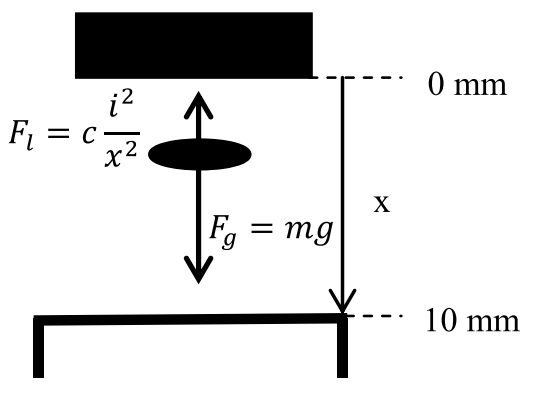

Fig. 3. Free body diagram of the magnetic levitation system.

implemented to filter the noisy signals at high frequencies. A current driver was used to control the current running through the coil, yielding a linear relation between voltage and current

$$
i=\frac{u}{67.83}
$$

where $u$ is the voltage $[\mathrm{V}]$ and $i$ is the current applied to the coil [A].

The electromagnet coil is a $24 \mathrm{~V}$ dc solenoid having the iron core replaced with an aluminum one. The suspended object is a permanent magnet and under this circumstances, it is preferred a material having a smaller magnetic permeability such that the magnet will not get stuck at the bottom of the coil. The levitating permanent disk magnet, made out of neodymiumferric-boric, weighs $1.79 \mathrm{~g}$, with a diameter of $10 \mathrm{~mm}$ and a height of $3 \mathrm{~mm}$. The coil has a diameter of $22 \mathrm{~mm}$, with a height of $13 \mathrm{~mm}$, and resistance of $95 \Omega$, being made out of 2600 turns, with each wire having a diameter of $0.18 \mathrm{~mm}$.

To model the magnetic levitation system, the free body diagram given in Fig. 3 is used, as well as electromagnetic and mechanical equations. The resulting net force acting on the permanent disk magnet, denoted $F_{\text {net }}$, is computed using Newton's law of motion while neglecting friction

$$
F_{\text {net }}=F_{g}-F_{l}=m g-c \frac{i^{2}}{x^{2}}
$$

where $F_{l}$ is the electromagnetic force (levitation force) [6] and $F_{g}$ is the gravitational force, $m$ is the mass of the permanent magnet $[\mathrm{kg}], g=9.81$ is the gravitational speed constant $\left[\mathrm{m} / \mathrm{s}^{2}\right], x$ is the distance $[m], \ddot{x}$ is the acceleration of the permanent magnet $\left[\mathrm{m} / \mathrm{s}^{2}\right]$, and $c$ is the magnetic force constant. The resulting net force is equal to

$$
F_{\text {net }}=m \ddot{x} \text {. }
$$

Replacing (22) into (21) leads to

$$
m \ddot{x}-m g+c \frac{i^{2}}{x^{2}}=0 .
$$

Denoting $f(x, \ddot{x}, i)=m \ddot{x}-m g+c\left(i^{2} / x^{2}\right)$, the equation in (23) is then linearized with respect to an equilibrium point $\left(x_{0}, i_{0}\right)$ by applying the Taylor series expansion

$$
\begin{aligned}
f(x, \ddot{x}, i)= & f\left(x_{0}, \ddot{x}_{0}, i_{0}\right)+\left.\frac{\partial f}{\partial x}\right|_{\left(x_{0}, \ddot{x}_{0}, i_{0}\right)}\left(x-x_{0}\right) \\
& +\left.\frac{\partial f}{\partial \ddot{x}}\right|_{\left(x_{0}, \ddot{x}_{0}, i_{0}\right)}\left(\ddot{x}-\ddot{x}_{0}\right)+\left.\frac{\partial f}{\partial i}\right|_{\left(x_{0}, \ddot{x}_{0}, i_{0}\right)}\left(i-i_{0}\right) .
\end{aligned}
$$

Using (23) into (24), leads to

$$
\begin{aligned}
f(x, \ddot{x}, i)= & \left.\left(-\frac{2 c i^{2}}{x^{3}}\right)\right|_{\left(x_{0}, \ddot{x}_{0}, i_{0}\right)}\left(x-x_{0}\right)+\left.m\right|_{\left(x_{0}, \ddot{x}_{0}, i_{0}\right)}\left(\ddot{x}-\ddot{x}_{0}\right) \\
& +\left.\left(\frac{2 c i}{x^{2}}\right)\right|_{\left(x_{0}, \ddot{x}_{0}, i_{0}\right)}\left(i-i_{0}\right) .
\end{aligned}
$$

Considering now the linearization point $\left(x_{0}, i_{0}\right)$, and denoting $\Delta i=i-i_{0}$ and $\Delta x=x-x_{0}$, (25) may be rewritten as

$$
f(x, \ddot{x}, i)=-\frac{2 c i_{0}^{2}}{x_{0}^{3}} \Delta x+m \Delta \ddot{x}+\frac{2 c i_{0}}{x_{0}^{2}} \Delta i=0 .
$$

Applying the Laplace transform to the linearized (26), leads to

$$
-\frac{2 c i_{0}^{2}}{x_{0}^{3}} \Delta x(s)+m s^{2} \Delta x(s)+\frac{2 c i_{0}}{x_{0}^{2}} \Delta i(s)=0
$$

which leads to the final transfer function of the mechanical subsystem

$$
\frac{\Delta x(s)}{\Delta i(s)}=-\frac{\frac{2 \mathrm{ci}_{0}}{x_{0}^{2}}}{m s^{2}-\frac{2 \mathrm{ci}_{0}^{2}}{x_{0}^{3}}} .
$$

For the electromagnetic subsystem, the relation between the voltage and the current in (20) is used, leading to the final transfer function for the magnetic levitation system

$$
\frac{\Delta x(s)}{\Delta u(s)}=-\frac{\frac{2 c i_{0}}{x_{0}^{2}}}{67.83\left(m s^{2}-\frac{2 c i_{0}^{2}}{x_{0}^{3}}\right)} .
$$

To determine the unknown parameter $c$ in (23), several experiments have been performed by placing the magnet at different distances from the coil and supplying the coil with a voltage large enough to raise the magnet. The experiments and resulting values for $c$, computed using (23), with $\ddot{x}=0$, are listed in Table I, in the Appendix. The final value for $c$ is computed as the mean value, averaging over the interval for $x_{0}$ between 3 and $6.5 \mathrm{~mm}$. In this interval, the value for $c$ does not vary much from the average computed value of $8.05 \times 10^{-5}$, and may be thus assumed as constant. For an equilibrium point chosen as $\left(x_{0}, i_{0}\right)=\left(4.2 \times 10^{-3} \mathrm{~m}, 49.8 \times 10^{-3} \mathrm{~A}\right)$, the transfer function in (29) becomes

$$
\frac{\Delta x(s)}{\Delta u(s)}=-\frac{6.1}{(s-70)(s+70)} .
$$

The transfer function in (30) is rewritten so as to be similar to $(1)$

$$
\frac{\Delta x(s)}{\Delta u(s)}=-\frac{1}{(s-70)(s+70)}
$$




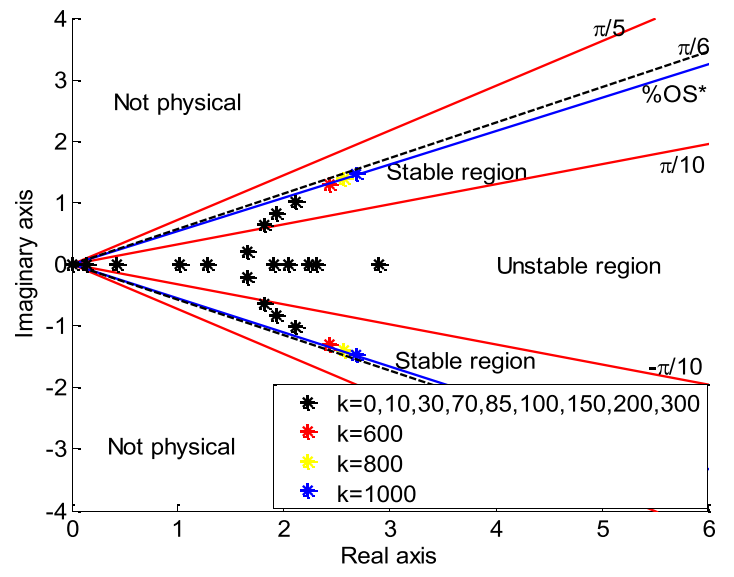

Fig. 4. Root locus for $n=5$ and $\lambda=0.2$.

where the gain 6.1 in (30) will be considered as part of the controller gain $k$, in (3). To tune a simplified stabilizing controller for the magnetic levitation system in (31), the stability analysis in Section II, as well as an overshoot requirement of $\% \mathrm{OS}^{*}=1 \%$ are employed. First, based on (17), $\zeta=0.8261$, while according to (18), $\alpha=0.97 \mathrm{rad}$. Finally, using (19), the following result is obtained:

$$
n>4.2 \text {. }
$$

Since $n$ is a positive integer, the minimum value that satisfies (32) is $n=5$, or $\lambda=0.2$, which is further used in the tuning of the parameter $k$ by plotting the root locus of (13). This is given in Fig. 4, for several values of $k$. It can be easily noted that for $k=600$, the root locus crosses the imposed \%OS* line.

For $k<600$, the closed-loop system will exhibit an oscillatory behavior, while for larger values of the gain $k$, the control effort will be increased, as also demonstrated in Figs. 5 and 6.

Fig. 5(a) and (b) shows the position of the disk, according to different reference step signals, and considering the openloop gain $k=600$ in comparison with $k=300$ and $k=1000$. As expected from Fig. 4, the lower the value of $k$, the closer the system is to the unstable region. The experimental results demonstrate this accurately, with high oscillatory behavior of the closed-loop system in the case of $k=300$, as indicated in Fig. 5(a). A higher value of the gain, $k=1000$, should result in roots of the characteristic equation closer to the secondary sheet of the Riemann surface.

This implies that the closed-loop system should exhibit an overdamped response. The experimental results given in Fig. 5(b) show that for $k=1000$, the magnetic disk tracks its reference position with less overshoot and less oscillations in comparison with the case when $k=600$. The required control signals are also given in Fig. 5(c) and (d). The results show that the larger the open-loop gain $k$, the larger the control effort. Thus, a value $k=600$ has been selected with the resulting FO controller indicated in (33). Considering that the process exhibits a 6.1 gain, the final FO controller gain is 98.5 , with the transfer function

$$
C(s)=-98.5\left(s^{0.8}+\frac{70}{s^{0.2}}\right) .
$$

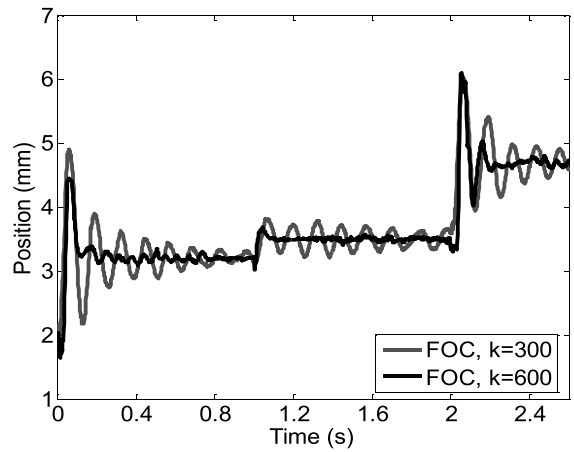

(a)

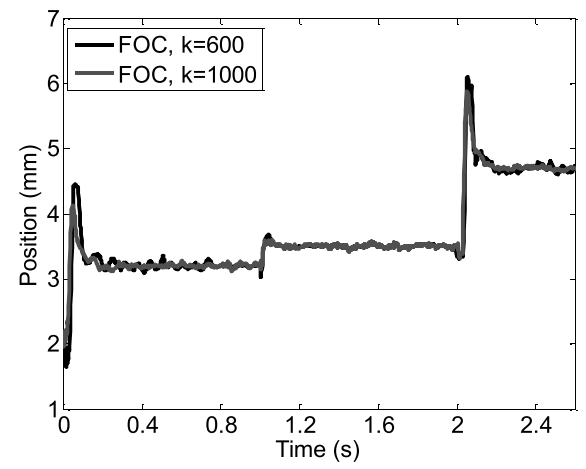

(b)

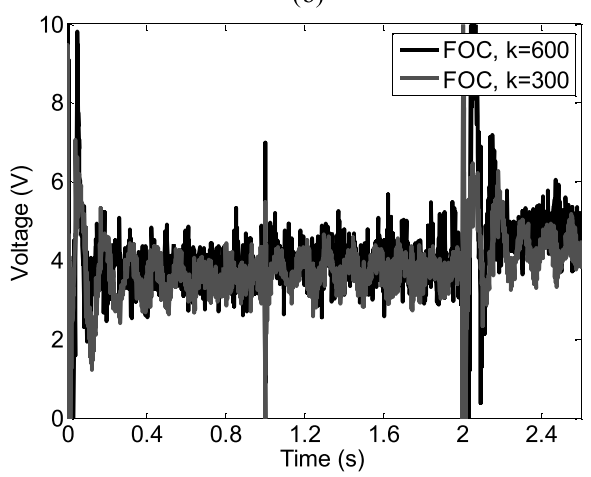

(c)

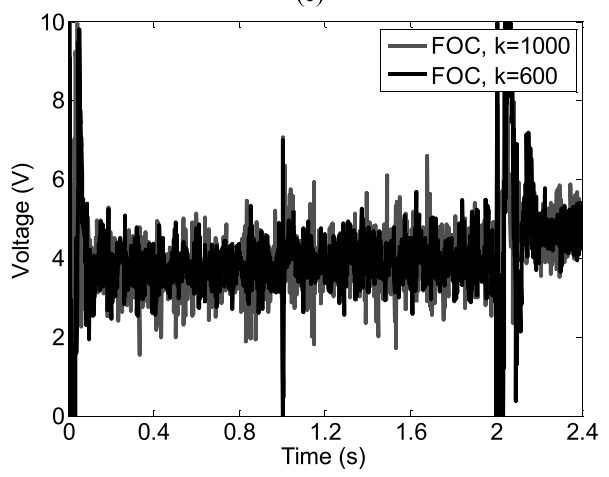

(d)

Fig. 5. Experimental results with the FO controller considering different open-loop gains. (a) Disk position for $k=600$ in comparison with $k=300$. (b) Disk position for $k=600$ in comparison with $k=1000$. (c) Control effort disk for $k=600$ (black line) in comparison with $k=300$ (gray line). (d) Control effort for $k=600$ (black line) in comparison with $k=1000$ (gray line).

To implement the FO controller, a digital approximation was derived using the recursive Tustin method, of ninth order, with a sampling time $T_{s}=2 \mathrm{~ms}$ [30]. 


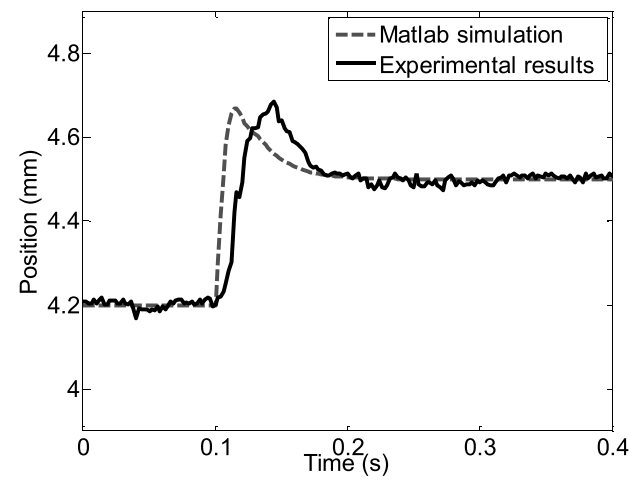

Fig. 6. Closed-loop simulation and experimental results considering a reference step change around the linearization point and the simplified FO controller.

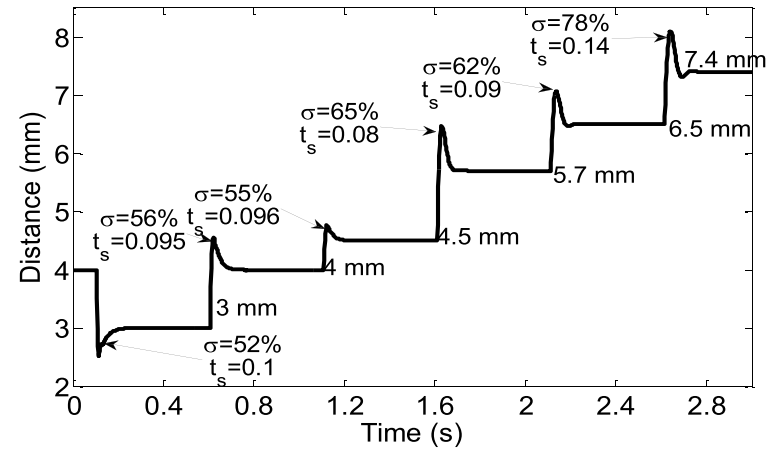

Fig. 7. Robustness simulation results considering several operating points and the simplified FO controller.

Fig. 6 shows the closed-loop simulation results, with the designed FO controller given in (33), in comparison with the experimental results. The reference position for the permanent disk changes from 4.2 to $4.5 \mathrm{~mm}$, thus close to the linearization point, $4.2 \mathrm{~mm}$. The simulation and experimental results in Fig. 6 are in reasonable agreement, both in terms of overshoot and settling time.

\section{Robustness Analysis}

To test the robustness of the designed controller, several reference step changes were considered, as indicated in Table II, in the Appendix. The parameter $c$ was also modified to fit the experimental data and computed values listed in Table I, rather than considering the averaged value. The corresponding transfer functions that approximate the behavior of the magnetic levitation system in each operation point are listed in Table II and have been used to obtain the simulation results given in Fig. 7. The simulation results in Fig. 7 show that around the linearization point, the overshoot and the settling time do not vary, with the overshoot $\sigma=55 \%$ and the settling time around $t_{s}=0.1 \mathrm{~s}$. When considering a wider operating point for the magnetic levitation system, with the position reference signal varying between 3 and $6.5 \mathrm{~mm}$ - the same interval used for computing the average value for the parameter $c$, the settling time does not modify much, however, the overshoot increases with nearly $20 \%$ (from $55 \%$ to $65 \%$, at a reference step change of $5.7 \mathrm{~mm}$ ). Outside this interval, the overshoot increases even more, as well as the settling time $\left(\sigma=78 \%\right.$ and $t_{s}=0.14 \mathrm{~s}$,

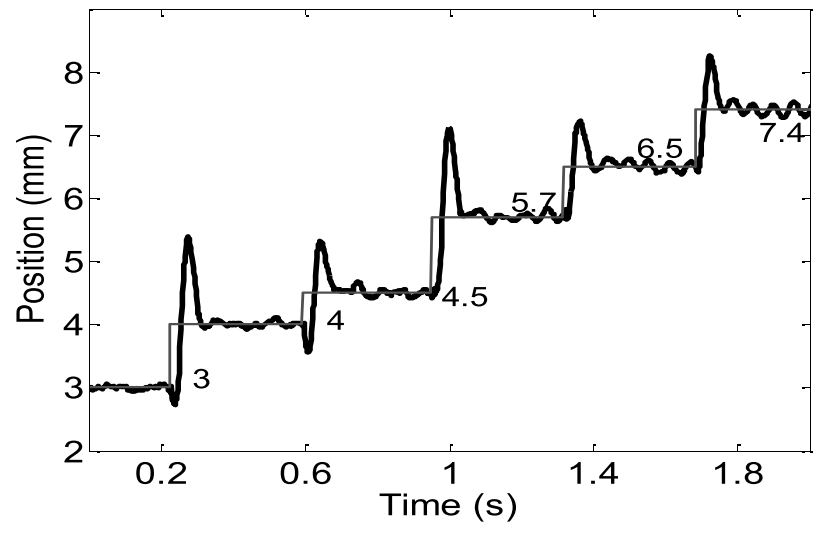

Fig. 8. Robustness experimental results considering several operating points and the simplified FO controller.

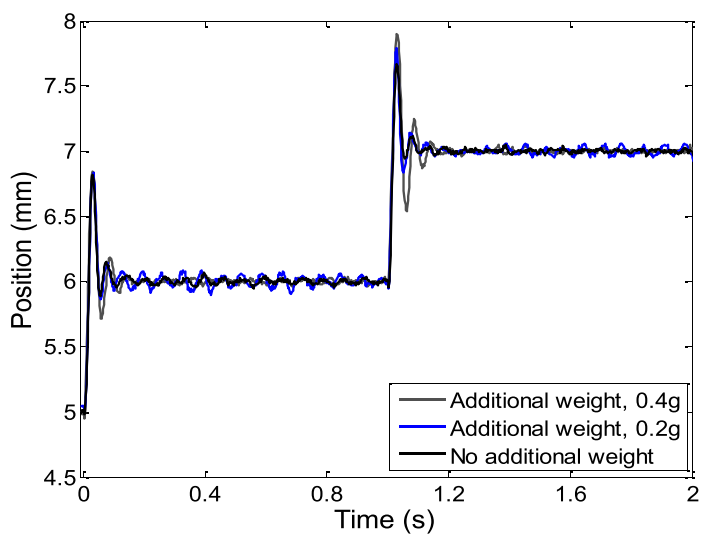

Fig. 9. Robustness experimental results considering a load placed on the permanent disk magnet and the simplified FO controller.

at a reference step change of $7.4 \mathrm{~mm}$ ). Nevertheless, the FO controller ensures the closed-loop stability of the magnetic levitation system.

The experimental results are given in Fig. 8. The designed controller stabilizes the magnetic levitation system, with a settling time $t_{s}=0.1 \mathrm{~s}$ in the linearization point, and a slight increase to $t_{s}=0.15 \mathrm{~s}$ at different operating points. The overshoot is higher, compared with the simulation results, but as expected from the nonlinear character of the magnetic levitation system.

Fig. 9 presents the staircase change in the reference signal, but considering different loading conditions, in which a supplementary weight was added to the permanent disk magnet: a low weight of $0.2 \mathrm{~g}$ and a medium weight of $0.4 \mathrm{~g}$, representing a $10 \%$ and $20 \%$, respectively, supplementary added weight. Apart from these additional loadings, modeling errors are also present in the experiment given in Fig. 9, as suggested by the results in Tables I and II in the Appendix. The experimental results in Fig. 9 show that the designed controller behaves robustly to these load changes and to the modeling errors associated to the change in the operating point. Comparing the experimental results considering the magnetic disk with no additional weight, the magnetic disk with an additional weight of 0.2 and $0.4 \mathrm{~g}$, respectively, the overshoot has a slight increase with increasing the weight, as well as a slight increase in the settling time. 


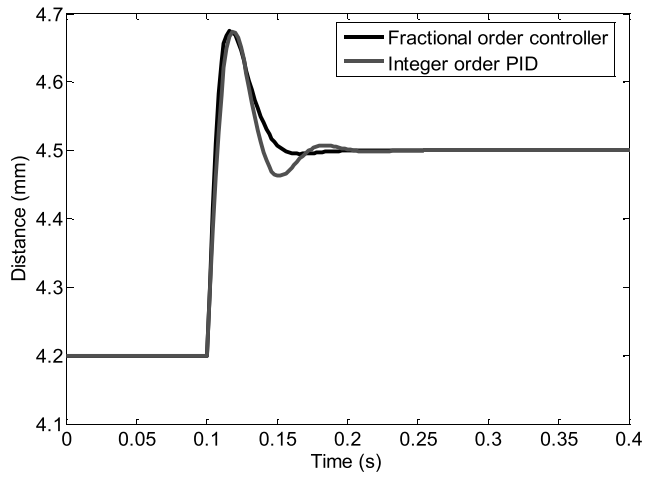

Fig. 10. Closed-loop simulation results considering a reference step change around the linearization point with FO controller and integer order PID.

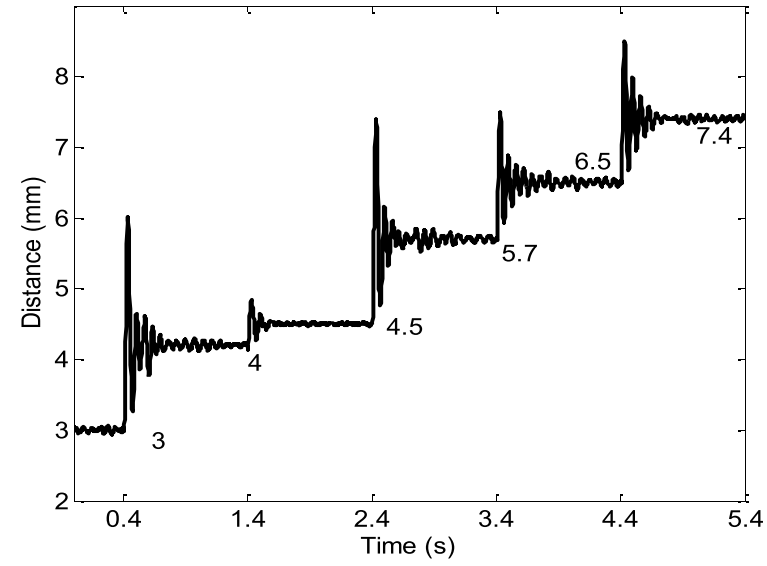

Fig. 11. Robustness experimental results considering several operating points and the integer order PID controller.

\section{COMPARison With A Simple InTEger ORDER PID CONTROLLER}

For comparison purposes, the equivalent integer order version of the controller given in (3) could be considered

$$
C_{\mathrm{PI}}(s)=-k \frac{(s+z)}{s}
$$

where $z$ is the open-loop pole of the process transfer function. The classical integer order PI controller in (34) would not be able to stabilize processes described as in (1). Thus, the classical integer order controller in (34) is altered to be able to meet the stabilizing requirement

$$
C_{\mathrm{PID}}(s)=-k \frac{(s+z)^{2}}{s} \text {. }
$$

Since the purpose of this brief has been to design simple controllers, the one given in (35) is the simplest form of a traditional PID controller that requires only one tuning parameter, the gain $k$. The tuning is performed using root locus analysis, such that the system will exhibit the same overshoot as the FO controller. Fig. 10 shows the closed-loop simulation results with the two proposed controllers: 1) the FO controller in (33) and 2) an integer order PID controller, as described in (35), with $k=27.1$. The PID controller achieves the same overshoot, with a slight
TABLE I

\begin{tabular}{|c|c|c|c|c|}
\hline No & $x_{0}(\mathrm{~m})$ & $i_{0}(\mathrm{~A})$ & $c$ & $\begin{array}{c}\text { Final value of } \\
c\end{array}$ \\
\hline 1 & $6.5 \cdot 10^{-3}$ & $68.1 \cdot 10^{-3}$ & $9.83 \cdot 10^{-5}$ & \multirow{5}{*}{$8.05 \cdot 10^{-5}$} \\
\hline 2 & $5.7 \cdot 10^{-3}$ & $62.2 \cdot 10^{-3}$ & $9.06 \cdot 10^{-5}$ & \\
\hline 3 & $4.5 \cdot 10^{-3}$ & $55.4 \cdot 10^{-3}$ & $7.12 \cdot 10^{-5}$ & \\
\hline 4 & $4.2 \cdot 10^{-3}$ & $49.8 \cdot 10^{-3}$ & $7.68 \cdot 10^{-5}$ & \\
\hline 5 & $3 \cdot 10^{-3}$ & $38.5 \cdot 10^{-3}$ & $6.55 \cdot 10^{-5}$ & \\
\hline
\end{tabular}

COMPUTATION OF THE PARAMETER $c$

TABLE II

MAGNETIC LEVITATION SySTEM TRANSFER FunCtions Corresponding TO A CERTAIN OpERATION POINT

\begin{tabular}{|c|c|c|c|}
\hline No & $\boldsymbol{x}_{\boldsymbol{\theta}}(\mathbf{m})$ & $\boldsymbol{c}$ & Transfer function \\
\hline 1 & $7.4 \cdot 10^{-3}$ & $10.6 \cdot 10^{-5}$ & $-\frac{2.94}{(\mathrm{~s}-51.5)(\mathrm{s}+51.5)}$ \\
\hline 2 & $6.5 \cdot 10^{-3}$ & $9.83 \cdot 10^{-5}$ & $-\frac{3.48}{(\mathrm{~s}-55)(\mathrm{s}+55)}$ \\
\hline 3 & $5.7 \cdot 10^{-3}$ & $9.06 \cdot 10^{-5}$ & $-\frac{4.13}{(\mathrm{~s}-58.7)(\mathrm{s}+58.7)}$ \\
\hline 4 & $4.5 \cdot 10^{-3}$ & $7.12 \cdot 10^{-5}$ & $-\frac{5.9}{(\mathrm{~s}-66)(\mathrm{s}+66)}$ \\
\hline 5 & $4.2 \cdot 10^{-3}$ & $7.68 \cdot 10^{-5}$ & $-\frac{6.1}{(\mathrm{~s}-68.3)(\mathrm{s}+68.3)}$ \\
\hline 6 & $3 \cdot 10^{-3}$ & $6.55 \cdot 10^{-5}$ & $-\frac{9.23}{(\mathrm{~s}-80.9)(\mathrm{s}+80.9)}$ \\
\hline
\end{tabular}

increase in the settling time, as compared with the FO controller.

The robustness simulation results considering several operating points given in Fig. 11 show a poorer closed-loop performance when compared with the results in Fig. 8 obtained with the proposed simplified FO controller. The magnetic levitation system experiences some increased oscillations and a larger overshoot when controlled with the PID in (35), especially away from the linearization point. The integer order PID provides a poorer robustness compared with the FO controller.

\section{CONCLUSION}

This brief has focused upon an alternative simpler solution to the stabilization of second-order unstable plants, which consists in a special type of an FO controller. The tuning procedure for this type of controller is very simple and it is based upon specifying the overshoot requirement and determining the controller parameters directly based on stability analysis. Such a tuning procedure has not been used before for unstable systems. A second advantage of the tuning procedure proposed in this brief, consists in the direct computation of the controller parameters, with no optimization routines required. A case study is also presented, consisting in a magnetic levitation system. The experimental results show that the designed FO controller ensures the stability of the closed-loop system in several operating points, as well as in the case of two different loads placed upon the permanent disk magnet. Although the 
robustness issue is not directly tackled in the tuning procedure, the designed FO controller proves to be robust to modeling uncertainties and nonlinearities. For comparison purposes, a simple integer order PID controller is designed to stabilize the magnetic levitation system and to ensure a similar settling overshoot as the FO controller. The experimental results show that in terms of robustness, the integer order PID controller has poorer performance compared with the proposed FO controller.

Further developments may include nonlinear predictive control techniques combined with fractional calculus, which can be applied directly on the nonlinear model of the magnetic levitation system, thus ensuring an improved robustness over the entire operating range.

\section{APPENDIX}

See Tables I and II.

\section{REFERENCES}

[1] R.-J. Wai and J.-D. Lee, "Robust levitation control for linear Maglev rail system using fuzzy neural network," IEEE Trans. Control Syst. Technol., vol. 17, no. 1, pp. 4-14, Jan. 2009.

[2] M. S. de Queiroz and S. Pradhananga, "Control of magnetic levitation systems with reduced steady-state power losses," IEEE Trans. Control Syst. Technol., vol. 15, no. 6, pp. 1096-1102, Nov. 2007.

[3] J. Kaloust, C. Ham, J. Siehling, E. Jongekryg, and Q. Han, "Nonlinear robust control design for levitation and propulsion of a Maglev system,' IEE Proc.-Control Theory Appl., vol. 151, no. 4, pp. 460-464, Jul. 2004.

[4] O.-S. Kim, S.-H. Lee, and D.-C. Han, "Positioning performance and straightness error compensation of the magnetic levitation stage supported by the linear magnetic bearing," IEEE Trans. Ind. Electron., vol. 50, no. 2, pp. 374-378, Apr. 2003

[5] K. S. Peterson, J. W. Grizzle, and A. G. Stefanopoulou, "Nonlinear control for magnetic levitation of automotive engine vales," IEEE Trans. Control Syst. Technol., vol. 14, no. 2, pp. 346-354, Mar. 2006.

[6] R. Morales, V. Feliu, and H. Sira-Ramírez, "Nonlinear control for magnetic levitation systems based on fast online algebraic identification of the input gain," IEEE Trans. Control Syst. Technol., vol. 19, no. 4, pp. 757-771, Jul. 2011.

[7] H. M. Gutierrez and P. I. Ro, "Magnetic servo levitation by slidingmode control of nonaffine systems with algebraic input invertibility," IEEE Trans. Ind. Electron., vol. 52, no. 5, pp. 1449-1455, Oct. 2005.

[8] Z. Yanhong, Z. Dean, Z. Jiansheng, Z. Zhongqiao, Z. Yiqin, and Z. Yongchun, "Robustness of improving active Maglev motorized spindle equilibrium position," TELKOMNIKA Indonesian J. Elect. Eng., vol. 11, no. 9, pp. 5161-5168, Sep. 2013.

[9] J. Xu and Y. Zhou, "A nonlinear control method for the electromagnetic suspension system of the Maglev train," J. Modern Transp., vol. 19, no. 3, pp. 176-180, Sep. 2011.

[10] H.-J. Shieh, J.-H. Siao, and Y.-C. Liu, "A robust optimal sliding-mode control approach for magnetic levitation systems," Asian J. Control, vol. 12, no. 4, pp. 480-487, Jul. 2010.

[11] R. L. Fittro and C. R. Knospe, "Rotor compliance minimization via $\mu$-control of active magnetic bearings," IEEE Trans. Control Syst. Technol., vol. 10, no. 2, pp. 238-249, Mar. 2002.
[12] A. Noshadi, J. Shi, W. S. Lee, P. Shi, and A. Kalam, "Genetic algorithm-based system identification of active magnetic bearing system: A frequency-domain approach," in Proc. 11th IEEE Int. Conf. Control Autom. (ICCA), Jun. 2014, pp. 1281-1286.

[13] R. Pizá, J. Salt, A. Sala, and Á. Cuenca, "Hierarchical triple-Maglev dual-rate control over a profibus-DP network," IEEE Trans. Control Syst. Technol., vol. 22, no. 1, pp. 1-12, Jan. 2014.

[14] C. Ionescu, J. T. Machado, and R. De Keyser, "Fractional-order impulse response of the respiratory system," Comput. Math. Appl., vol. 62, no. 3 , pp. 845-854, 2011.

[15] L. Zhu and C. R. Knospe, "Modeling of nonlaminated electromagnetic suspension systems," IEEE/ASME Trans. Mechatronics, vol. 15, no. 1, pp. 59-69, Feb. 2010

[16] A. Oustaloup, J. Sabatier, and P. Lanusse, "From fractional robustness to CRONE control," Fractional Calculus Appl. Anal., vol. 2, no. 1, pp. 1-30, 1999

[17] I. Podlubny, "Fractional-order systems and $P I^{\lambda} D^{\mu}$ controllers," IEEE Trans. Autom. Control, vol. 44, no. 1, pp. 208-214, Jan. 1999.

[18] Y. Luo, Y. Q. Chen, C. Y. Wang, and Y. G. Pi, "Tuning fractional order proportional integral controllers for fractional order systems," J. Process Control, vol. 20, no. 7, pp. 823-831, 2010.

[19] E.-H. Dulf, C.-I. Pop, and F.-V. Dulf, "Fractional calculus in ${ }^{13} \mathrm{C}$ separation column control," Signal, Image Video Process., vol. 6, no. 3, pp. 479-485, 2012.

[20] C. Copot, A. Burlacu, C. M. Ionescu, C. Lazar, and R. De Keyser, "A fractional order control strategy for visual servoing systems," Mechatronics, vol. 23, no. 7, pp. 848-855, 2013.

[21] J.-Y. Cao and B.-G. Cao, "Design of fractional order controller based on particle swarm optimization," Int. J. Control, Autom. Syst., vol. 4 no. 6, pp. 775-781, 2006

[22] C. A. Monje, Y. Chen, B. M. Vinagre, D. Xue, and V. Feliu-Batlle, Fractional-order Systems and Controls: Fundamentals and Applications. London, U.K.: Springer-Verlag, 2010.

[23] C. I. Muresan, S. Folea, G. Mois, and E. H. Dulf, "Development and implementation of an FPGA based fractional order controller for a DC motor," Mechatronics, vol. 23, no. 7, pp. 798-804 2013.

[24] I. Kheirizad, A. A. Jalali, and K. Khandani, "Stabilization of all-pole unstable delay systems by fractional-order [PI] and [PD] controllers," Trans. Inst. Meas. Control, vol. 35, no. 3, pp. 257-266, 2013.

[25] M. S. Tavazoei and M. Haeri, "Stabilization of unstable fixed points of chaotic fractional order systems by a state fractional PI controller," Eur J. Control, vol. 14, no. 3, pp. 247-257, 2008.

[26] L.-Y. Chang and H.-C. Chen, "Tuning of fractional PID controllers using adaptive genetic algorithm for active magnetic bearing system," WSEAS Trans. Syst., vol. 8, no. 1, pp. 158-167, 2009.

[27] J. Zhong and L. Li, "Fractional-order system identification and proportional-derivative control of a solid-core magnetic bearing," ISA Trans., vol. 53, no. 4, pp. 1232-1242, 2014.

[28] R. Caponetto, G. Dongola, L. Fortuna, and I. Petras, Fractional Order Systems: Modeling and Control Applications. Singapore: World Scientific, 2010.

[29] A. G. Radwan, A. M. Soliman, A. S. Elwakil, and A. Sedeek, "On the stability of linear systems with fractional-order elements," Chaos, Solitons Fractals, vol. 40, no. 5, pp. 2317-2328, 2009.

[30] Y. Q. Chen and K. L. Moore, "Discretization schemes for fractional-order differentiators and integrators," IEEE Trans. Circuits Syst. I, Fundam. Theory Appl., vol. 49, no. 3, pp. 363-367, Mar. 2002. 